\title{
Solved and unsolved questions in the non-rigid Earth nutation study
}

\author{
C. L. Huang
}

Shanghai Astronomical Observatory, Chinese Academy of Sciences, 80 Nandan Rd., Shanghai 200030, China. Email: clhuang@shao.ac.cn

\begin{abstract}
At the IAU 26th GA held in Prague in 2006, a new precession model (P03) was recommended and adopted to replace the old one, IAU1976 precession model. This new P03 model is to match the IAU2000 nutation model that is for anelastic Earth model and was adopted in 2003 to replace the previous IAU1980 model. However, this IAU2000 nutation model is also not a perfect one for our complex Earth, as stated in the resolution of IAU nutation working group. The Earth models in the current nutation theories are idealized and too simple, far from the real one. They suffer from several geophysical factors: the an-elasticity of the mantle, the atmospheric loading and wind, the oceanic loading and current, the atmospheric and oceanic tides, the (lateral) heterogeneity of the mantle, the differential rotation between the inner core and the mantle, and various couplings between the fluid outer core and its neighboring solids (mantle and inner core). In this paper, first we give a very brief review of the current theoretical studies of non-rigid Earth nutation, and then focus on the couplings near the core-mantle boundary and the inner core-outer core boundary, including the electro-magnetic, viscous, topographic, and gravitational couplings. Finally, we outline some interesting future studies.
\end{abstract}

Keywords. reference systems, standards, magnetic fields, Earth, methods: analytical

\section{Introduction}

Nutation is the periodic motion of a conceptual axis, called the Celestial Intermediate Pole (CIP), or previously, the Celestial Ephemerids Pole (CEP), in space with periods between 2 days and 18.6 years as seen from space. To replace the previous IAU1980 model, the new nutation model adopted by the IAU in 2000 is the so-called MHB2000 model (Mathews et al. 2002) that is for anelastic Earth model. At the IAU 26th GA held in Prague in 2006, a new precession model, P03 (Capitaine et al. 2003), as well as corresponding definition and terminology, was recommended and adopted to replace the old one, IAU1976 precession model, to match the IAU2000 nutation model (see also contribution by Capitaine (this volume) for a review of definition and realization of the celestial intermediate reference system).

Observation on nutation Space geodetic techniques especially VLBI have developed very fast in the past 3 decades and have played the most important role in observation of nutation, while LLR have also contributed a lot to the long period terms (like 18.6 yr), and GPS may have also helped to determine short period nutations $(<9 \mathrm{~d})$. Generally speaking, nutations can be determined by observation with uncertainties at the level of 40 and $10 \mu \mathrm{as}$ for 18.6-year and other terms respectively. For determination of rotational normal modes, their uncertainties can reach approximately $0.1,0.1$, and 5 day for Chandler wobble $(\mathrm{CW})$, free core nutation $(\mathrm{FCN})$, and free inner core nutation (FICN) respectively. Besides these high precisions, another achievement is that they can provide the nutation solution in very short intervals, say 1 hour. However, there are still some discussions on the problems of VLBI observation on nutation: for example, the VLBI 
network geometry and observation strategy, radio source structure, and the software for solving EOP, etc.

Steps to study non-rigid earth nutation (NREN) Generally, in order to calculate a nonrigid earth nutation, we first need a rigid earth nutation (REN) model that is usually derived from celestial mechanics, by the torque, Hamilton or tidal potential method with ephemerides of the Sun, the Moon and planets. Second, from the equations of infinitesimal elastic gravitational motion for a rotating, slightly elliptical earth, Poisson equation, stress-strain relation equation, a set of boundary conditions, and a given earth model that gives the internal profiles of density, elastic coefficients and so on, we can get two products: one is the normal modes, the other is the so-called earth transfer function (ETF) that represents the response of a non-rigid earth (NRE) to outer force or excitation. Third, convolving the REN series with this ETF, we get a NREN series. Finally, incorporating the effects of other geophysical factors including surface fluids (atmosphere and ocean) and dynamic processes inside the Earth (eg. various coupling at core-mantle-boundary $(\mathrm{CMB})$ and inner-core-boundary (ICB)), with correction afterward by torque or angular momentum methods, or via boundary conditions in the second step, we get a new nutation model of a more realistic earth.

Theoretical approaches and models Generally, we can classify available methods for calculating non-rigid earth nutation into 3 categories: (1)numerical integration or displacement field approach used by Smith, Wahr, Dehant, Schastok, Huang, and else; (2)angular momentum approach used in SOS theory by Sasao et al. and MHB model by Mathews et al.; (3)Hamiltonian approach that was first used in REN theory, and then developed by Getino \& Ferrándiz et al. and applied to NREN study.

On the one hand, all the above theoretical nutation models are very comparable to each other although they use different approaches; on the other hand, one apparent 'advantage' of MHB2000 over the other models is that the observed differences in the principal nutation terms (mainly the retro-annual (-1yr) term) with respect to VLBI observations are reduced by accounting for the effects of electro-magnetic coupling (EMC) of the FOC to the mantle and the SIC (Buffett et al., 1992, 2002), in which the coupling constants at $\mathrm{CMB}$ and $\mathrm{ICB}$ and related compliance parameters are fitted to the VLBI nutation observations.

\section{Phenomena}

Comparing between theoretical nutation models and observation, or between different nutation models, the main problematic terms become obvious: 18.6yr \& -1yr nutations, and the out-of-phase (op) components of some terms as related to dissipation. The second phenomenon is about the global dynamic ellipticity $(\mathrm{H})$ that will be discussed later. The third concerns FCN that depends very intensively on the physical and dynamical properties near CMB. From observation, its period is about 430 day, while from PREM, it is about 460 day. Moreover, with more and more observation data accumulated, it tends to agree that the amplitude and period of FCN vary with time. Is it true, and why do they change? The biggest effect of these phenomena is on -1yr nutation due to strong resonance. The fourth phenomenon concerns FICN, which is also important for geophysicist as it reflects the physics and dynamics near the ICB. But since it is very faint, the question "is the 'observed' FICN a true one?" is still uncertain.

There are many celestial mechanical and geophysical (both inside and outside the Earth) factors in computing theoretical nutation. We can classify them into 4 groups: (1)the direct and indirect gravitational forces from the sun, the moon and the planets; (2)excitations of the surface geophysical fluids; (3)properties of the Earth itself, i.e. the 
earth model; (4)dynamical processes inside the earth, including various couplings between its 3 layers, and large scale convection in the mantle, and so on.

REN model: All the REN models can be regarded as perfect compared with the NREN models. They can be consistent with each other in $10 \mu$ as or better, although there are still some unresolved issues, such as general relativistical effects, and all 2nd-order (in)direct effects.

Surface geophysical fluids: In regard to contributions from surface geophysical fluids, for both oceans and atmosphere, and including both load (or pressure) and motion terms, one can use effective angular momentum method, torque method, or direct integration method by introducing an outer surface boundary condition (Huang et al., 2001). They influence mostly 18.6yr, 1yr and 0.5yr nutations, as well as prograde annual nutation

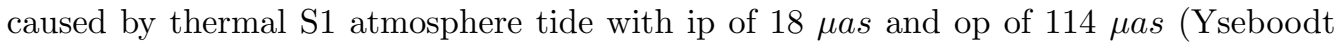
et al., 2002). Generally, the diurnal atmospheric excitation is less than half that required to explain the observed FCN amplitude (Lambert, 2006). In addition to previous studies focusing on resonance at FCN, Dehant et al. (2005) show that resonance at FICN induced by surface geophysical fluids only induces a very small signal, with a maximum of $20 \mu a \mathrm{~s}$ at 880-day prograde term.

Earth model: Next, although seismology has presented many earth models, the most often used one in nutation study is still the PREM model. There are many shortcomings in PREM, such as (1)it is a 1-dimension model depending on the spherical radii only; (2)the Earth is assumed to be in hydro-static equilibrium (HSE); (3)the medium is isotropic; and (4)how to treat the ocean layer is also a major problem. However, currently we do not have a choice. For example, using the PREM model, we can get the ellipticity of the CMB and the period of FCN at about -458 days. However, if we assume there is a deviation from HSE near the CMB, and increase the equator's radius at the CMB by 400 meter, then we get the FCN at -432 days, which is closer to observation (Huang et al., 2001).

Global dynamic flattening $(H)$ : By definition, $H=\frac{C-\frac{1}{2}(A+B)}{C}$, where A, B and $\mathrm{C}$ are the three principal moments of inertia of the Earth. Therefore $H$ depends on the density distribution inside the Earth, and is related to the lunar-solar main precession, main nutation, tilt-over-mode (TOM) and so on. Precession observations give $H_{o b s} \approx 1 / 305.5$, while it is approximately $1 / 308.8$ at the first order accuracy from the PREM model. This $1.1 \%$ difference has stimulated many interesting discussions. Using a more precise potential theory to third-order accuracy in HSE, Liu \& Huang (2008, this Volume) recalculate the geometrical flattening $(f)$ profile of the Earth interior from PREM and obtain $H_{P R E M}=1 / 308.5$. However, After replacing the homogenous outermost crust and oceanic layers in PREM with some real surface layers data, such as topography+ocean, topography+ocean+bathmetry+upper crust data, or CRUST2.0 model, down to depth of $5.615 \mathrm{~km}, 10.376 \mathrm{~km}, 70.137 \mathrm{~km}$ respectively, they obtain $1 / H=318.14,320.22,310.70$ respectively. These results deviate from the observed value more than $H_{P R E M}$ and verify the isostasy theory indirectly. They may imply that 'positive' effects arising from, for example, mantle circulation associated with the density anomalies may be larger than what was discussed before.

\section{Dynamical processes inside the earth}

There may exist 4 kinds of angular momentum or torque coupling near the CMB and ICB: gravitational, electro-magnetic (EM), viscous, and topographic. And there may also be deviation from HSE. 
Gravitational coupling: This mechanism can be used to fill all main gaps between the theoretical model and observation (eg., Jault \& LeMouel, 1989), but the key parameters used are too arbitrary.

EM coupling: As mentioned above, it is used by Buffett et al. in their MHB model to explain the op component of -1yr nutation, but it is open to question. For example, taking a direct numerical integration approach, Huang et al.(2006) show that, even using the same values of EM properties as the MHB model did, possible contribution of EM coupling to -1yr nutation is only about one-tenth of what is required. The authors of the MHB model themselves also realized that the contribution of EM coupling maybe not so significant as they declared before, so they and other colleagues divert their interest to viscous coupling at CMB (Mathews \& Guo, 2005; Deleplace \& Cardin, 2006; Buffett \& Christensen 2007).

Viscous coupling: In order to fill the gap for -1yr nutation. the effective viscosity of the core fluid is required to be at the level of $0.03 \mathrm{~m}^{2} / \mathrm{s}$. However, if based on laboratory and physical consideration of the fluid viscosity, the eddy viscosity is smaller than $10^{-4} \mathrm{~m}^{2} / \mathrm{s}$ and therefore may be also too small for the -1yr nutation.

Topographic torque at CMB: It is also a potential mechanism for explaining the dissipation in the -1yr nutation. It is related to core angular momentum exchange and pressure at the CMB. It also depends very intensively on topography at the CMB. For example, Wu \& Wahr (1997) concluded that the non- $Y_{2}^{0}$ parts of the topography of the CMB within $3.5,4-5$, or $6-7 \mathrm{~km}$ (rms) may contribute to the $-1 \mathrm{yr}$ nutation by $0.55,0.77$ or 2 mas respectively. However, the seismology tomography data shows that the topography at the $\mathrm{CMB}$ is smaller than $2 \mathrm{~km}$, and the above results seem to lack support from seismology. Therefore it is still a difficult, challenging and controversial topic but cannot be completely ruled out (see also Mound \& Buffett, 2003).

\section{Some other discussions}

Lateral heterogeneity: There was some research on the effects of lateral heterogeneity on earth tides based on a 3D earth model. For example, it may change the gravity tidal admittance $\delta_{M 2}$ by up to $0.5 \%$ (Wang, 1991), and the Love number $k_{M 2}$ by about $0.19 \%$ (Li et al.,1996). But so far there is not any study in nutation, maybe due to the difficulty in theoretical work.

$2^{n} d$ order theory and terms: Although there are some theoretical studies of the effects of the truncated $2^{n} d$ order terms on nutation (Huang, 2001; Van Hoolst \& Dehant, 2002; Rogister \& Rochester, 2004), there is not any numerical result of its direct effect so far.

Lambert (2006) (see also Lambert \& Mathews, 2006) calculated zonal tides indirect effects that are $37 \mu a s$ and $1 \mu a s$ for the $\Delta \psi$ and $\Delta \varepsilon$ of $18.6 y r$ nutation and $-518 \mu a s / \mathrm{c}$ for precession in $\psi$. Folgueira et al.(2007) and Dehant et al.(2007) also discussed tidal Poisson terms, which are periodic in amplitude linearly dependent on time, and showed that it may change the nutation by approximately several tens $\mu a s$ or several $\mu a s$ for a very long period (10467.6 years) term.

Differential rotation: The possible differential rotation of the inner core with respect to the mantle $\left(\Delta \Omega_{I C-M}\right)$ may contribute a little to 18.6 -yr nutation. For example, if $\Delta \Omega_{I C-M}=1^{\circ} / y r$, the biggest change will be $\delta \varepsilon_{i p}^{18.6 y} \approx 0.01$ mas (Huang \& Dehant, 2002). Although it is very small, it may be detectable by nutation observation in future.

\section{Short remarks}

From the above brief review, we see that nutation study suffers mostly from the rough information of the earth model (mostly from the CMB to the outer surface) and the 
dynamical processes near the CMB. Therefore, precise nutation observation provides another way to study the earth interior besides seismology.

\section{Acknowledgements}

This work is supported by NSFC (10773025/10633030), CAS(KJCX2-YW-T13), and Science \& Technology Commission of Shanghai Municipality (06DZ22101/06ZR14165).

\section{References}

Buffett, B. A. 1992, J. Geophys. Res., 97(B13), 19581

Buffett, B. A., Mathews, P. M., \& Herring, T. A. 2002, J. Geophys. Res., 107(B4), DOI: 10.1029/2000JB000056

Buffett, B. A., \& Christersen, U. R. 2007, Geophys. J. Int., 171, 145

Capitaine, N., Chapront, J, Lambert, S., \& Wallance, P. T. 2003, Astron. \& Astrophys., 400, 1145, doi: 10.1051/0004-6361:20030077

Dehant, V., de Viron, O., \& Greff-Lefftz, M. 2005, Astron. \& Astrophys., 438, 1149, doi: 10.1051/0004-6361:20042210

Dehant, V., Folgueira, M., Rambaux, N., \& Lambert, S. B. 2007, Proc. IUGG XXIVth GA (in press)

Deleplace, B. \& Cardin, P. 2006, Geophys. J. Int., 167, 557

Folgueira, M., Dehant, V., Lambert, S. B., \& Rambaux, N. 2007, Astron. \& Astrophys., 469, 1197, doi: 10.1051/0004-6361:20066822

Huang, C. L. 2001, Earth, Moon, and Planets, 84, 125

Huang, C. L., Jin, W. J., \& Liao, X. H. 2001, Geophys. J. Int., 146, 126

Huang, C. L. \& Dehant, V. 2002, in: N. Capitaine (ed.), Proceedings of Journées 2001, 20

Huang, C. L., Dehant, V., Liao, X. H., de Viron, O., \& Van Hoolst, T. 2006, in: IAU XXVIth GA Abstract book, 404

Jault, D., Le Mouël, J. L. 1989, Geophys. \& Astrophys. Fluid Dyn., 48(4), 273, DOI:10.1080/03091928908218533

Lambert, S. 2006, Astron. \& Astrophys., 457, 717, doi: 10.1051/0004-6361:20065813

Lambert, S., \& Mathews, P. M. 2006, Astron. E Astrophys., 453, 363, doi: 10.1051/00046361:20054516

Li, G. Y., Peng, L. H., \& Xu, H. Z. 1996, Acta Geophys. Sinica, 39, 672

Mathews, P. M., Herring, T. A., \& Buffett, B. A. 2002, J. Geophys. Res., 107(B4), DOI: 10.1029/2001JB000390

Mathews, P. M., \& Guo, J. Y. 2005, J. Geophys. Res., 110(B), DOI: 10.1029/2003JB002915

Mound, J. E. \& Buffett, B. A. 2003, J. Geophys. Res., 108(B7), DOI: 10.1029/2002JB002054

Rogister, Y. \& Rochester, M. 2004, Geophys. J. Int., 159, 874

Van Hoolst, T., \& Dehant, V. 2002, Phys. Earth Planet.Inter., 134, 17

Wang, R. J. 1991, Tidal deformation on a rotating, spherically asymmetric, visco-elastic and laterally heterogeneous Earth (Frankfurt am Main: Peter Lang)

Wu, X. P. \& Wahr, J. 1997, Geophys. J. Int., 128, 18

Yseboodt, M., de Viron, O., Chin, T. M., \& Dehant, V. 2002, J. Geophys. Res., 107(B2), DOI: 10.1029/2000JB000042 